This item was submitted to Loughborough's Research Repository by the author.

Items in Figshare are protected by copyright, with all rights reserved, unless otherwise indicated.

\title{
Vibration measurements using continuous scanning laser vibrometry: velocity sensitivity model experimental validation
}

PLEASE CITE THE PUBLISHED VERSION

http://dx.doi.org/10.1088/0957-0233/14/6/310

PUBLISHER

(c) Institute of Physics Publishing

\section{LICENCE}

CC BY-NC-ND 4.0

\section{REPOSITORY RECORD}

Halkon, Ben J., S.R. Frizzel, and Steve Rothberg. 2019. "Vibration Measurements Using Continuous Scanning Laser Vibrometry: Velocity Sensitivity Model Experimental Validation". figshare.

https://hdl.handle.net/2134/3320. 
This item was submitted to Loughborough's Institutional Repository by the author and is made available under the following Creative Commons Licence conditions.

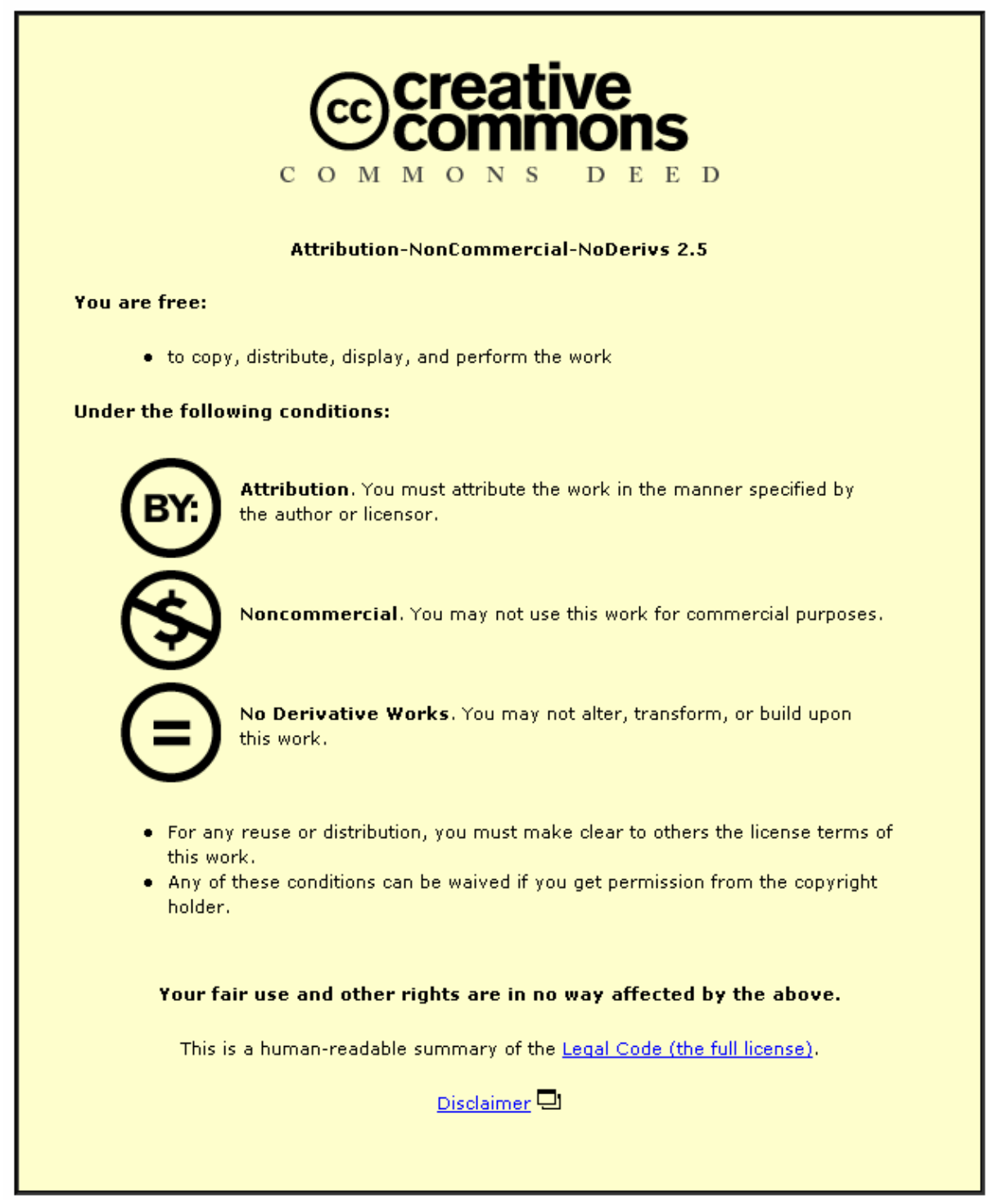

For the full text of this licence, please go to: http://creativecommons.org/licenses/by-nc-nd/2.5/ 


\section{VIBRATION MEASUREMENTS USING CONTINUOUS \\ SCANNING LASER VIBROMETRY: VELOCITY \\ SENSITIVITY MODEL EXPERIMENTAL VALIDATION}

(CONTINUOUS SCANNING LASER VIBROMETRY: VELOCITY

SENSITIVITY MODEL EXPERIMENTAL VALIDATION)

B. J. Halkon, S. R. Frizzel and S. J. Rothberg

Wolfson School of Mechanical and Manufacturing Engineering

Loughborough University, Loughborough, Leicestershire, LE11 3TU, U.K.

e-mail: b.j.halkon@lboro.ac.uk, tel: +44 (0) 1509 227589, fax: +44 (0) 1509227648 


\begin{abstract}
This paper builds on a previous study in which the theoretical description of the velocity sensed by a single laser beam incident in an arbitrary direction on a rotating target undergoing arbitrary vibration was extended to continuous scanning Laser Vibrometer measurements on targets with flexible cross-sections. The velocity sensitivity model was written in terms of either laser beam orientation angles or deflection mirror scan angles, with the latter found to be most useful for continuous scanning applications.

The model enables the prediction of the Laser Vibrometer output for any measurement configuration on any target. The experimental validation presented in this paper confirms that additional components appear in rotating target measurements that are associated with both the scanning system configuration and any misalignment between the scanning system and target rotation axes. This paper will show how use of the velocity sensitivity model enables the vibration engineer to make LDV measurements with confidence.
\end{abstract}

KEYWORDS: Laser Doppler Vibrometry, scanning, tracking, velocity sensitivity, vibration measurement, rotating machinery. PACS: 06.30.Gv, 07.60.-j, 43.40.+s. 


\section{INTRODUCTION}

The principle of Laser Doppler Vibrometry (LDV) relies on the detection of a Doppler shift in the frequency of coherent light scattered by a moving target, from which a timeresolved measurement of the target velocity is obtained. The Laser Vibrometer is now well established as an effective non-contact alternative to the use of a traditional contacting vibration transducer. Laser Vibrometers are technically well suited to general application but offer special benefits where certain measurement constraints are imposed, for example by the context, which may demand high frequency operation, high spatial resolution or remote transducer operation, or by the structure itself, which may be hot, light or rotating. Measurements on such structures are often cited as important applications of LDV [1] and scanning LDV is of particular current interest. Commercial scanning Laser Vibrometers incorporate two orthogonally aligned mirrors, and can operate point by point, in continuous scanning mode $[2,3,4]$ or in tracking mode [5] in which the probe laser beam remains fixed on a single point on a target such as a rotating bladed disc. Throughout the remainder of this paper, "scanning" LDV refers to operation in continuous scanning mode rather than point-by-point.

In a previous publication [6], a recently developed theoretical description of the velocity measured by a single laser beam incident in an arbitrary direction on a rotating target undergoing arbitrary motion [7] was extended to the particularly challenging application of scanning Laser Vibrometer measurements on rotating targets with flexible crosssections. The original velocity sensitivity model was reformulated to make use of mirror scan angles rather than laser beam orientation angles, which is especially useful as it is these that the user would seek to control in a real scanning system. In both cases, it was shown to be straightforward to accommodate time dependency in the angles thereby enabling the prediction of the instrument output when operating in scanning mode. The 
advanced applications of circular scans on rotating targets and small-scale circular and conical scans on non-rotating targets were investigated as a means of illustrating the effectiveness of the model for the analysis of actual scan configurations. In particular, the origin of an additional component that occurs at $2 \mathrm{x}$ scan frequency in a circular scanning measurement on a rotating target was shown to be associated with the dual mirror configuration. The velocity sensitivity model can also be used effectively to predict the result of any translational and/or angular misalignment between the scanning system and target rotation axes and this important issue is discussed in detail for the first time in this paper.

Previous studies have presented and experimentally validated mathematical descriptions of the velocity sensed in Laser Vibrometer measurements on rotating targets undergoing radial [8] and torsional [9] vibration, demonstrating an important cross-sensitivity to motions perpendicular to the intended measurement. The general velocity sensitivity model was applied to, and validated for, single [7] and multiple [10] beam measurements for arbitrary six degree of freedom vibrations. This model has predicted additional components that specifically occur in scanning Laser Vibrometer measurements on rotating targets and their experimental validation is the particular focus of this paper.

The influence of noise generated by the laser speckle effect [11] is also of particular importance in scanning Laser Vibrometer measurements. This and the effect of offset between the laser beam and the deflection mirrors are also discussed for the first time in this paper. Finally, a prediction of the Laser Vibrometer output is compared with a real circular scanning axial vibration measurement on a rotating target. 


\section{THEORETICAL VELOCITY SENSITIVITY MODEL REVIEW}

\subsection{VELOCITY MEASURED BY A DUAL MIRROR SCANNING LASER}

\section{VIBROMETER}

With reference to Figure 1, a typical scanning measurement is performed by the introduction of two orthogonally aligned mirrors, separated by some distance $d_{S}$, into the laser beam path. The scanning system optical axis is defined as being the line along which the laser beam is directed towards the target when there is "zero" beam deflection. In this particular configuration, the scanning system and target reference frames are collinear such that the scanning system optical axis lies on the $z$ axis of the target reference frame.

The mirror scan angles, $\theta_{S x}$ and $\theta_{S y}$, are defined as positive if in an anticlockwise sense and "zero" when the resulting laser beam direction, described by the unit vector $\hat{b}$, is along the scanning system optical axis ( $z$ axis). Expressing the direction of each mirror surface as a unit vector, it is possible to calculate $\hat{b}$ in terms of $\theta_{S x}$ and $\theta_{S y}$ [6]:

$$
\hat{b}=\left[\sin 2 \theta_{S x}\right] \hat{x}-\left[\cos 2 \theta_{S x} \sin 2 \theta_{S y}\right] \hat{y}+\left[\cos 2 \theta_{S x} \cos 2 \theta_{S y}\right] \hat{z} .
$$

This important equation defines the incident laser beam direction for any combination of deflection mirror scan angles and can be used to derive an expression for the velocity measured, $U_{m}$, in a scanning Laser Vibrometer measurement on a rotating target of flexible cross-section undergoing 6 degree-of-freedom vibration [6]:

$$
\begin{aligned}
U_{m} & \left.=\sin 2 \theta_{S x} \mid \dot{x}_{r}\left(P_{0}\right)+\dot{x}_{f}(P)\right\rfloor \\
& -\cos 2 \theta_{S x} \sin 2 \theta_{S y}\left\lfloor\dot{y}_{r}\left(P_{0}\right)+\dot{y}_{f}(P)\right\rfloor \\
& \left.+\cos 2 \theta_{S x} \cos 2 \theta_{S y} \mid \dot{z}_{r}\left(P_{0}\right)+\dot{z}_{f}(P)\right\rfloor,
\end{aligned}
$$

in which $\dot{x}_{f}(P), \dot{y}_{f}(P), \dot{z}_{f}(P)$ are the vibration velocity components in the $x, y, z$, directions due to cross-section flexibility (the point $\mathrm{P}$ represents the instantaneous point 
of incidence of the laser beam on the arbitrarily deformed target) and $\dot{x}_{r}\left(P_{0}\right), \dot{y}_{r}\left(P_{0}\right)$, $\dot{z}_{r}\left(P_{0}\right)$ are the resultant vibration velocity components in the $x, y, z$ directions due to rigid body vibration (the point $\mathrm{P}_{0}$ represents the corresponding point on the undeformed target), given by:

$$
\begin{aligned}
& \dot{x}_{r}\left(P_{0}\right)=\dot{x}-\left(\dot{\theta}_{z}+\Omega\right)\left(y_{0}-y\right)+\left(\dot{\theta}_{y}-\Omega \theta_{x}\right)\left(z_{0}-z\right), \\
& \dot{y}_{r}\left(P_{0}\right)=\dot{y}+\left(\dot{\theta}_{z}+\Omega\right)\left(x_{0}-d_{S} \tan 2 \theta_{S x}-x\right)-\left(\dot{\theta}_{x}+\Omega \theta_{y}\right)\left(z_{0}-z\right)
\end{aligned}
$$

and

$$
\dot{z}_{r}\left(P_{0}\right)=\dot{z}+\left(\dot{\theta}_{x}+\Omega \theta_{y}\right)\left(y_{0}-y\right)-\left(\dot{\theta}_{y}-\Omega \theta_{x}\right)\left(x_{0}-d_{S} \tan 2 \theta_{S x}-x\right) .
$$

Here, $\dot{x}, \dot{y}, \dot{z}$ and $x, y, z$ are the translational vibration velocities and displacements, $\dot{\theta}_{x}, \dot{\theta}_{y}, \dot{\theta}_{z}$ and $\theta_{x}, \theta_{y}$ are the angular vibration velocities and displacements and $\Omega$ is the total rotation frequency of the target.

The original derivation [7] involved the definition of an arbitrary known point $\left(x_{0}, y_{0}\right.$, $z_{0}$ ), which lies along the line of the laser beam. With reference to Figure 1, it can be seen that, for the dual mirror scanning arrangement, there is no single point from which the laser beam appears to originate. Here, the most convenient known point to choose is the incidence point of the beam on the $y$ deflection mirror, which scans back and forth along the mirror rotation axis. The known point $x$ coordinate is, therefore, a function of the $x$ mirror scan angle and, as such, $x_{0}$ should include this oscillatory component, which is given by [6]: $-d_{S} \tan 2 \theta_{S x}$. In the interest of clarity, however, $x_{0}$ is slightly redefined to exclude this component, which is then shown explicitly in equations $(3 b \& c)$.

Equation (2) is important as it allows the user to predict the sensitivity of a scanning Laser Vibrometer measurement for any combination of mirror scan angles on any target and readily accommodates time dependent mirror scan angles where scanning profiles 
result. This is discussed in the following section, which describes application of the model to circular scanning measurements on rotating targets.

\subsection{CIRCULAR SCANNING}

\subsubsection{Typical and Corrected Mirror Scan Angles}

A "circular" scan can be performed by using cosine and sine mirror drive signals of the form:

$$
\theta_{S x}=-\Theta_{S x} \cos \left(\Omega_{S} t+\phi_{S}\right)
$$

and

$$
\theta_{S y}=\Theta_{S y} \sin \left(\Omega_{S} t+\phi_{S}\right)
$$

where $\Theta_{S x}$ and $\Theta_{S y}$ are the $x$ and $y$ mirror scan amplitudes and $\Omega_{S}$ and $\phi_{S}$ are the scan angular frequency and initial phase. The use of equal scan amplitudes, e.g.:

$$
\Theta_{S x}=\Theta_{S y}=0.5 \tan ^{-1}\left(\frac{r_{S}}{z_{0}}\right),
$$

where $r_{S}$ is the intended scan radius and $z_{0}$ is the stand-off distance between the target and the Laser Vibrometer, results in a slightly elliptical shape in the scan trajectory. For a typical scanning configuration $\left(d_{S}=50 \mathrm{~mm}\right.$ and $\left.z_{0}=1 \mathrm{~m}\right)$ this corresponds to an error in the intended scan radius of 5\% [6]. This error can be overcome to an extent by accounting for the difference between the target to $x$ mirror and target to $y$ mirror distances and employing corrected drive signals with unequal amplitudes, e.g.:

$$
\Theta_{S x}=0.5 \tan ^{-1}\left(\frac{r_{S}}{z_{0}+d_{S}}\right)
$$

and

$$
\Theta_{S y}=0.5 \tan ^{-1}\left(\frac{r_{S}}{d_{S}}\right) .
$$


In this case, the error in the scan radius is reduced to less than $0.05 \%$ (for the same configuration), which may be advantageous in some cases [6]. Generally more important, however, is the influence that the variation in laser beam orientation during scanning has on the Laser Vibrometer measurement and this will be discussed in the following section.

\subsubsection{Circular scans for rotating targets}

Use of equation (2) allows prediction of the measured velocity in a scanning Laser Vibrometer measurement and it also shows how additional components can occur when performing measurements on rotating targets. Substituting equations (4a\&b) and (5) into equation (2) immediately results in an expression for the measured velocity in an equal amplitude drive signal "circular" scan on a non-vibrating, rotating target [6]. In this "no target vibration, no misalignment" case, significant additional information exists in the measured Laser Vibrometer output at twice the scan frequency. This component has been observed previously [5] but without full explanation until recently $[6]$.

Modified configurations, such as the corrected drive signal case, can be modelled just as easily, in this case by substituting equations $(4 a \& b)$ and $(6 a \& b)$ into equation (2) to give a corresponding expression for the measured velocity [6]. Despite the substantial improvement in the scan profile, significant additional information still exists at twice the scan frequency, with typical levels only 5\% lower, for this particular combination of $d_{S}=50 \mathrm{~mm}$ and $z_{0}=1 \mathrm{~m}$, than for the equal amplitude case. The prediction of such additional components in the Laser Vibrometer output demonstrates the value of the velocity sensitivity model very clearly - it enables the vibration engineer to predict the instrument output for any measurement configuration on any target and therefore make Laser Vibrometer measurements with confidence. 
The model can also be used to examine the effects of misalignment between the target and scanning system axes and this important aspect of real applications will be explored in detail for the first time in the next section of this paper.

\section{SCANNING SYSTEM AND TARGET ROTATION AXIS}

\section{MISALIGNMENT}

The model can be used to predict the effect that translational and/or angular misalignment between the scanning system and target rotation axes has on the measurement, which is particularly useful since small misalignments are inevitable. As illustrated in Figure 2, translational misalignment can be accounted for in the model by adding the constants $x_{0 m}$ and $y_{0 m}$ to the intended known point $x$ and $y$ coordinates. Similarly, angular misalignment is represented by adding the constants $\theta_{x m}$ and $\theta_{y m}$ to the angular vibration displacement parameters. Setting the flexible and rigid vibration components to zero in equation (2) enables the measured velocity (ideally zero, of course) to be predicted for this "no target vibration, arbitrary misalignment" case. Making use of equations (3a,b\&c), equation (2) can be used to show the influence of misalignments on the measured velocity:

$$
\begin{aligned}
& \frac{U_{m}}{\Omega}=\cos 2 \theta_{S x} \cos 2 \theta_{S y}\left[\theta_{x m} x_{0 m}+\theta_{y m} y_{0 m}\right] \\
& +\sin 2 \theta_{S x}\left\lfloor y_{0 m}+\theta_{x m}\left(z_{0}+d_{S} \cos 2 \theta_{S y}\right)\right]-\cos 2 \theta_{S x} \sin 2 \theta_{S y}\left\lfloor x_{0 m}-\theta_{y m} z_{0}\right\rfloor \\
& -d_{S} \sin 2 \theta_{S x} \sin 2 \theta_{S y} .
\end{aligned}
$$

Substitution for $\theta_{S x}$ and $\theta_{S y}$ using equations (4a\&b) and (5) or equations $(4 \mathrm{a} \& \mathrm{~b})$ and $(6 a \& b)$ immediately results in a full expression for the velocity measured in either an equal amplitude or corrected amplitude mirror drive signal circular scan. In either case, significant additional information exists in the measured Laser Vibrometer signal at DC, $1 \mathrm{x}$ and $2 \mathrm{x}$ scan frequency, as illustrated in Figure 3 (equal amplitude case). The 
amplitude of the component at $2 \mathrm{x}$ scan frequency is identical to that which occurs in the no misalignment case discussed in section 2.2.2, demonstrating that this component occurs only as a result of the dual mirror scanning configuration. This can be confirmed by rearrangement and inspection of equation (7) and is intuitive when small angle approximations can be applied. Such small angle approximations are appropriate in the experimental investigation that was undertaken to validate the prediction of these additional components using the model and this is the subject of the following section.

\section{EXPERIMENTAL VALIDATION}

\subsection{EXPERIMENTAL ARRANGEMENT}

The scanning system used was custom built using a Polytec OFV323 Laser Vibrometer and a pair of GSI Lumonics M3 galvanometers. Each galvanometer can drive the mirror angular displacement by $\pm 15^{\circ}$ mechanical $\left( \pm 30^{\circ}\right.$ optical). A two-channel signal generator was used to generate the equal amplitude cosine and sine functions necessary to perform a "circular" scan. The galvos are mounted relative to the Laser Vibrometer in the same manner as shown schematically in Figure 1 and Figure 2, which is equivalent to the arrangement employed in both the Ometron Type 8330 and Polytec PSV300 commercial scanning Laser Vibrometers.

The target used was a small $(\varnothing 30 \mathrm{~mm} \times 5 \mathrm{~mm})$, aluminium disc of rigid cross-section mounted to a DC motor. The target rotation frequency was controlled using a stable DC power supply and measured using a Polytec OFV400 Rotational Laser Vibrometer.

\subsection{EXPERIMENTAL PROCEDURE}

Substituting for $\theta_{S x}$ and $\theta_{S y}$ using equations (4a\&b) and (5) and using small angle approximations enables equation (7) to be re-written for an "equal amplitude mirror drive signal, no target vibration, arbitrary misalignment" scan: 


$$
\begin{aligned}
\frac{U_{m}}{\Omega} & =\left[\theta_{x m} x_{0 m}+\theta_{y m} y_{0 m}\right] \\
& +\frac{r_{S}}{z_{0}}\left[y_{0 m}+\theta_{x m}\left(z_{0}+d_{S}\right)\right] \cos \left(\Omega_{S} t+\phi_{S}\right)-\frac{r_{S}}{z_{0}}\left[x_{0 m}-\theta_{y m} z_{0}\right] \sin \left(\Omega_{S} t+\phi_{S}\right) \\
& -\frac{d_{S} r_{S}^{2}}{2 z_{0}{ }^{2}} \sin 2\left(\Omega_{S} t+\phi_{S}\right)
\end{aligned}
$$

Clearly the corresponding expression for a "corrected amplitude mirror drive signal, no target vibration, arbitrary misalignment" scan can be formed by substituting for $\theta_{S x}$ and $\theta_{S y}$ using equations $(4 \mathrm{a} \& \mathrm{~b})$ and $(6 \mathrm{a} \& \mathrm{~b})$ and using small angle approximations. In either case, the components at $\mathrm{DC}, 1 \mathrm{x}$ and $2 \mathrm{x}$ scan frequency dominate the measurement, as previously discussed and illustrated in Figure 3 (equal amplitude case). As shown in equation (8), the amplitude of the component at $2 \mathrm{x}$ scan frequency is insensitive to variations in misalignment and, as such, it is possible to perform the experimental validation of this element of the velocity sensitivity model separately.

\subsubsection{Dual Mirror Effects}

Since the amplitude of the $2 \mathrm{x}$ component is a function of the perpendicular mirror separation, $d_{S}$, as well as the scan radius, $r_{S}$, and the stand-off distance, $z_{0}$, the scanning system hardware used in this validation included the facility to vary $d_{S}$ from $30 \mathrm{~mm}$ to 50mm. Figure 4a (equal amplitude case) and Figure $4 \mathrm{~b}$ (corrected amplitude case) show comparisons between the predicted and measured amplitude of the $2 \mathrm{x}$ component for a series of measurement configurations. In both cases, each solid line represents the theoretical prediction of $U_{m} / \Omega$ vs. $r_{S}$, with the plotted points representing the corresponding measured values. The theoretical prediction shows good correlation with the measured data for both equal amplitude and corrected amplitude mirror drive signal “circular" scans. 


\subsubsection{Misalignment Effects}

As shown in equation (8), translational and angular misalignments mostly influence the $1 \mathrm{x}$ additional measurement component. The scanning system used in this validation incorporated the facility to vary $x_{0 m}, y_{0 m}$ and $\theta_{y m}$ to enable further validation of the theory.

Whilst the "no target vibration" condition is relatively straightforward to achieve in the laboratory by taking care with target selection, the "no misalignment" condition is not. Small but inevitable initial misalignment between the scanning system and target rotation axes results in a significant component at $1 \mathrm{x}$ scan frequency.

Figure 5 shows a comparison between the predicted and measured amplitude of the additional component at the scan frequency for a series of measurements in which only the translational misalignment in the $x$ direction, $x_{0 m}$, was varied. The broken line represents the theoretical prediction of measured velocity per unit rotation frequency, $U_{m} / \Omega$, for varying $x_{0 m}$ and the seven data points represent the corresponding series of measured values. The difference between these two sets of data is significant and is due to the initial misalignments, which are unknown, difficult to control and cannot be measured directly. In the absence of a means to measure the initial misalignments directly, the chosen way to proceed is to use the velocity sensitivity model as a basis for a particular hypothesis. Experimentally demonstrating that the hypothesis holds can then be taken as validation of the model. The basis of this hypothesis is to use the velocity sensitivity model to obtain estimates for the initial misalignments after a series of measurements have been made in which one of the controllable misalignments is varied.

Introducing specific unknown initial misalignment parameters, $x_{0 m u}, y_{0 m u}, \theta_{x m u}$ and $\theta_{y m u}$, into equation (8) and evaluating at $1 \mathrm{x}$ scan frequency results in: 


$$
\begin{aligned}
\left(\frac{\left.U_{m}\right|_{\omega=\Omega_{S}}}{\Omega}\right)^{2} & =\left(\frac{r_{S}}{z_{0}}\right)^{2}\left[\left(y_{0 m}+y_{0 m u}\right)+\left(\theta_{x m}+\theta_{x m u}\right)\left(z_{0}+d_{S}\right)\right]^{2} \\
& +\left(\frac{r_{S}}{z_{0}}\right)^{2}\left[\left(x_{0 m}+x_{0 m u}\right)-\left(\theta_{y m}+\theta_{y m u}\right) z_{0}\right]^{2} .
\end{aligned}
$$

Making $x_{0 m}$ the variable misalignment parameter with $y_{0 m}, \theta_{x m}$ and $\theta_{y m}$ set to zero, enables this equation to be rearranged into a form that is useful for identifying the unknown misalignment parameters:

$$
\begin{aligned}
\left(\frac{\left.U_{m}\right|_{\omega=\Omega_{S}}}{\Omega}\right)^{2}-\left(\frac{r_{S}}{z_{0}} x_{0 m}\right)^{2} & =\left(\frac{r_{S}}{z_{0}}\right)^{2}\left[\left(y_{0 m u}+\left(z_{0}+d_{S}\right) \theta_{x m u}\right)^{2}+\left(x_{0 m u}-z_{0} \theta_{y m u}\right)^{2}\right] \\
& +2\left(\frac{r_{S}}{z_{0}}\right)^{2} x_{0 m}\left(x_{0 m u}-z_{0} \theta_{y m u}\right) .
\end{aligned}
$$

The first part of the hypothesis is that a plot of the terms of the left hand side of this expression against $x_{0 m}$ will result in a good fit to a straight line and this is confirmed in Figure 6a. Estimates of $\left(x_{0 m u}-z_{0} \theta_{y m u}\right)$ and $\left(y_{0 m u}+\left(z_{0}+d_{S}\right) \theta_{x m u}\right)$ based on gradient and intercept are then possible but, whilst the value of the former term can be substituted directly into equation (9), the value of the latter term is ambiguous in sign and, as such, cannot be used.

Following a similar procedure but in this case making $y_{0 m}$ the variable misalignment parameter with $x_{0 m}, \theta_{x m}$ and $\theta_{y m}$ set to zero enables equation (9) to be rearranged as follows:

$$
\begin{aligned}
\left(\frac{\left.U_{m}\right|_{\omega=\Omega_{S}}}{\Omega}\right)^{2}-\left(\frac{r_{S}}{z_{0}} y_{0 m}\right)^{2} & =\left(\frac{r_{S}}{z_{0}}\right)^{2}\left[\left(y_{0 m u}+\left(z_{0}+d_{S}\right) \theta_{x m u}\right)^{2}+\left(x_{0 m u}-z_{0} \theta_{y m u}\right)^{2}\right] \\
& +2\left(\frac{r_{S}}{z_{0}}\right)^{2} y_{0 m}\left(y_{0 m u}+\left(z_{0}+d_{S}\right) \theta_{x m u}\right) .
\end{aligned}
$$

As illustrated in Figure $6 \mathrm{~b}$, this part of the hypothesis is further supported by the strength of the fit to a straight line. The gradient of this plot can be used to obtain an 
unambiguous estimate of $\left(y_{0 m u}+\left(z_{0}+d_{S}\right) \theta_{x m u}\right)$. Note that in this case the estimate of $\left(x_{0 m u}-z_{0} \theta_{y m u}\right)$ is ambiguous in sign.

The angular misalignment parameters, $\theta_{x m}$ and $\theta_{y m}$ can also be used in the same manner to obtain estimates of the unknown initial misalignments. It is not, however, possible to estimate the individual unknown initial misalignments using any combination of the data generated by introducing further translational or angular misalignments. Fortunately this is not necessary, as only the estimates of the combinations of parameters are required to update the theoretical prediction of the amplitude of the $1 \mathrm{x}$ scan frequency component.

The second part of the hypothesis is that by substituting the estimated values of the unknown initial misalignment quantities into equation (9), a much improved theoretical prediction of the $1 \mathrm{x}$ scan frequency component will be obtained. These updated predicted amplitudes are also shown in Figure 5 (solid line) and they exhibit strong correlation with the measured data points, supporting this part of the hypothesis.

Figure 7 and Figure 8 show the corresponding comparisons between the predicted and measured amplitudes of the 1x scan frequency component for a series of measurements in which $y_{0 m}$ and $\theta_{y m}$, respectively, were varied, further supporting this part of the hypothesis. Again, in each case, the initial predicted amplitudes (broken line) are significantly different to the measured data points. The updated prediction (solid line) displays strong correlation in the $y_{0 m}$ case and reasonable correlation in the $\theta_{y m}$ case $\left(\theta_{y m}\right.$ was less easy to control in this particular experimental arrangement).

Figure 9 shows a comparison between the updated prediction (solid line) and measured amplitudes of the additional component at $1 \mathrm{x}$ scan frequency for a series of measurements in which the target rotation frequency, $\Omega$, was varied. Again, there is good correlation between the two data sets. 
The final part of the hypothesis is that estimates of the unknown initial misalignments should be reasonable given the care taken in ensuring that the scanning system and target rotation axes were aligned. From the data presented in Figure $6 a \& b$ and estimating that the amplitudes of the unknown initial translational misalignments to be each $1 \mathrm{~mm}$, the unknown initial angular misalignments would be in the region $0.3^{\circ}$ to $0.4^{\circ}$ (worst case). The nature of the experimental configuration used in this validation is such that these small angular misalignments are quite reasonable, supporting the final part of the hypothesis. The strength of the hypothesis at each of the three stages is taken as a validation of the velocity sensitivity model for prediction of this significant $1 \mathrm{x}$ scan frequency additional component.

\subsubsection{Laser Speckle Effects}

The velocity sensitivity model does not include the effects of laser speckle but, since laser speckle is such an important phenomenon in scanning LDV, it will be discussed in this section. Speckle noise is introduced into the Laser Vibrometer signal whenever the collected speckle pattern changes during the course of the measurement [11]. Clearly, such speckle pattern changes will occur in a circular scanning measurement due to the difference between the scan frequency and target rotation frequency and/or due to distortions in the scan trajectory.

An appreciation of the speckle noise phenomenon leads to the interesting notion that configuring a scanning Laser Vibrometer to perform a tracking measurement (i.e. setting the scan frequency equal to the target rotation frequency) should lead to a significant reduction in the speckle noise level since changes in the collected speckle pattern are minimised. A series of measurements were carried out to investigate this notion. Figure 10 shows the total spectral RMS speckle noise vs. the scan frequency, with the target rotation frequency nominally $50 \mathrm{~Hz}$. The $1 \mathrm{x}$ and $2 \mathrm{x}$ components have 
been eliminated from this calculation since their amplitudes are dominated by the mechanisms described earlier in sections 4.2.1\& 4.2.2. Without elimination of these two components, estimation of the signal noise due only to laser speckle effects would not be possible.

The solid and broken lines represent the mean and mean \pm standard deviation, respectively, for a number of tests. This data clearly shows that there is a general reduction in the speckle noise level as the scan frequency approaches the rotation frequency and a significant reduction when the scan frequency is the same as the rotation frequency, i.e. in a tracking Laser Vibrometer measurement.

\subsubsection{Doppler Shifts from the Scanning Mirrors}

In the development of the velocity sensitivity model for scanning Laser Vibrometer measurements [6], it was assumed that there was no Doppler shift due to the relative motion of the mirrors. This requires that each mirror rotation axis was in the plane of the respective mirror reflective surface and that the laser beam was reflected at a point on this axis. Neither assumption, however, is satisfied in a real scanning system and in some situations the resulting influence on the Laser Vibrometer signal should be accounted for when interpreting measured data.

The effect is characterised by an additional Doppler shift on the laser beam with a frequency equal to the scan frequency and a level dependent upon the mirror rotation angular frequency. During the course of this particular experimental validation, however, this additional component was at least an order of magnitude smaller $(1.4 \mathrm{~mm} / \mathrm{s}$ for a $50 \mathrm{~mm}, 10 \mathrm{~Hz}$ scan $)$ than that due to scanning system and target rotation axis misalignment and it was therefore considered insignificant. 


\section{AXIAL VIBRATION MEASUREMENT}

The main objective of this paper so far has been to validate the velocity sensitivity model for scanning measurements, in particular to verify the prediction of additional components that occur at integer multiples of the scan frequency in measurements on rotating targets. In this section, the velocity sensitivity model will be used to predict the form of the Laser Vibrometer output in a circular scanning measurement on a rotating target of rigid cross-section undergoing a medium severity axial vibration.

Figure 11 shows the velocity measured in a non-scanning Laser Vibrometer measurement on a non-rotating target undergoing $40 \mathrm{~Hz}, 10 \mathrm{~mm} / \mathrm{s}$ (nominal) axial vibration. This straightforward measurement constitutes a baseline for the scanning measurements, illustrating the vibration peak at $40 \mathrm{~Hz}$, as well as the genuine low level harmonic distortions at $80 \mathrm{~Hz}$ and $120 \mathrm{~Hz}$. The underlying instrument noise floor contains peaks at $50 \mathrm{~Hz}$ and $100 \mathrm{~Hz}$, which are caused by electrical interference and, as such, are present in all measured spectra presented in this section.

Figure 12 shows the velocity measured in a scanning Laser Vibrometer measurement on a non-rotating target undergoing nominally the same axial vibration. As discussed in section 4.2.3, scanning the laser beam causes speckle pattern motions during the course of the measurement that manifest themselves clearly in the spectrum at integer multiples of scan frequency. The peak at $1 \mathrm{x}$ scan frequency is at a higher level as a result of the additional Doppler shifts from the scanning mirrors, as discussed in section 4.2.4. Despite these sources of additional velocity content, the spectrum represents a respectable measurement of the $40 \mathrm{~Hz}$ axial vibration and its harmonic distortion.

In the case of a scanning Laser Vibrometer measurement on a rotating target, illustrated in Figure 13, the introduction of target motion into the measurement should result in a general difference in the resulting speckle noise. For a $10 \mathrm{~Hz}$ scan on a target rotating at 
$20 \mathrm{~Hz}$, a speckle pattern repeat of $10 \mathrm{~Hz}$ is still expected but, since the rotation frequency is only nominally $20 \mathrm{~Hz}$ in this measurement, the speckle pattern does not repeat perfectly at $10 \mathrm{~Hz}$. Despite this, higher speckle noise levels can be seen at approximately integer multiples of $10 \mathrm{~Hz}$ in the real measurement. More importantly, Figure 13 shows significantly higher velocity levels at $1 \mathrm{x}$ and $2 \mathrm{x}$ scan frequency, due to the arbitrary initial misalignment and the dual mirror configuration, respectively, and most importantly of all, a respectable measurement of the axial vibration.

The, now experimentally validated, theory presented in sections 2 and 3 enables the user to predict the form of the measured velocity and this is shown in Figure 14 for a typical scanning arrangement with typical misalignment values. The harmonic distortion of the axial vibration is not included in this velocity prediction.

As discussed in section 4.2.2, it is not possible to quantify the initial unknown misalignments in a real measurement, nor is it generally possible to repeat the test procedure used to estimate them. It should be possible, however, to estimate typical expected values from the measurement configuration and therefore produce an order of magnitude prediction for the $\mathrm{DC}$ and $1 \mathrm{x}$ additional components. The $2 \mathrm{x}$ additional component can be estimated with a high degree of accuracy in a real measurement, since it is insensitive to misalignment. As shown in Figure 14, small sidebands are present in the predicted instrument output at the vibration frequency $\pm \Omega_{S}$. These are caused by the misalignments and are generally very low-level components, below the instrument noise floor and therefore insignificant.

In the measurement discussed in this section, the scan frequency, rotation frequency and vibration level and frequency were chosen such that the additional measurement content did not constitute a significant source of measurement ambiguity. In a real measurement such control is obviously not available and, for example, if the axial vibration level 
were lower or at a different frequency, then the significance of the additional content might be greater as it would be less straightforward to distinguish genuine vibration peaks from additional content peaks. In such a situation, changing the scan frequency might be useful since the frequency of additional content peaks would change in sympathy whilst the frequency of the vibration peaks would remain constant.

The information presented in this paper provides the user with the ability to predict the additional components that occur in real scanning Laser Vibrometer measurements and thereby anticipate the form of the resulting spectra, of which Figure 13 is a typical example. Such measurements can then be interpreted with confidence.

\section{CONCLUSIONS}

The use of Laser Vibrometers incorporating some form of manipulation of the laser beam orientation, typically using two orthogonally aligned mirrors, has become increasingly popular in recent years. This paper has validated elements of a recently developed theoretical model for the prediction of the velocity sensed by a Laser Vibrometer in continuous scanning mode.

The velocity sensitivity model was presented in such a manner that it can be straightforwardly implemented to predict the Laser Vibrometer output for any system configuration and any combination of mirror scan angles. In the case of circular scanning measurements on rotating targets, the occurrence of significant additional components at DC and integer multiples of the scan frequency was shown to be due to both the dual mirror arrangement and misalignment between the scanning system and target rotation axes.

Experimentation showed that the additional component at $2 \mathrm{x}$ scan frequency was easy to validate but the additional component at $1 \mathrm{x}$ scan frequency was more difficult to 
validate. This component is due to translational and angular misalignment between the scanning system and target rotation axes and small but inevitable initial misalignments dramatically affect the measured amplitude. A hypothesis was proposed in which the model itself was used to predict the unknown initial misalignments and then used to improve the prediction of the $1 \mathrm{x}$ scan frequency component with further controlled misalignment. Confirmation of each part of the hypothesis was taken as the validation of the model for the prediction of misalignment-related additional components.

The influence of noise generated by the laser speckle effect and the effect of offset between the laser beam and either one or both of the deflection mirror rotation axes were highlighted as sources of additional measured velocity in scanning LDV. Finally, a prediction of the Laser Vibrometer output was compared with a real circular scanning measurement on a rotating target undergoing a medium severity axial vibration. The correlation between predicted and measured data was strong and the usefulness of the model in enabling confident interpretation of the measurement was thereby confirmed. 


\section{ACKNOWLEDGEMENT}

The authors would like to acknowledge the support of the Engineering and Physical

Sciences Research Council who are funding this project and who also funded the ENSURE project that supported Stuart Frizzel. 


\section{REFERENCES}

1. N. A. HALLIWELL in D. C. WILliAMS (ed.) 1993 Optical Methods in Engineering Metrology (London: Chapman \& Hall), 179-211. Chapter 6 - Laser Vibrometry.

2. P. SRIRAM, J. I. CRAIG and S. HANAGUD 1990 The International Journal of Analytical and Experimental Modal Analysis 5(3), 155-167. A scanning laser Doppler vibrometer for modal testing.

3. A. B. STANBRIDGE and D. J. EWINS 1999 Mechanical Systems and Signal Processing 13(2), 255-270. Modal testing using a scanning laser Doppler vibrometer.

4. A. B. STANBRIDGE and D. J. EWINS 1995 Transactions of ASME - Design Engineering Technical Conference 3(B), 1207-1213. Modal testing of rotating discs using a scanning LDV.

5. P. CASTELLINI and N. PAONE 2000 Review of Scientific Instruments 71(12), 4639-4647. Development of the tracking laser vibrometer: performance and uncertainty analysis.

6. B. J. HALKON and S. J. ROTHBERG 2003 Measurement Science and Technology 14(3), 382-393. Vibration measurements using continuous scanning laser vibrometry: theoretical velocity sensitivity analysis with applications.

7. J. R. BELL and S. J. ROTHBERG 2000 Journal of Sound and Vibration 237(2), 245-261. Laser vibrometers and contacting transducers, target rotation and six degreeof-freedom vibration: what do we really measure?

8. S. J. ROTHBERG and N. A. HALLIWELL 1994 Transactions of ASME-Journal of Vibration and Acoustics 116(3), 326-331. Vibration measurements on rotating machinery using laser Doppler velocimetry. 
9. T. J. MILES, M. LUCAS, N. A. HALLIWELL and S. J. ROTHBERG 1999 Journal of Sound and Vibration 226(3), 441-467. Torsional and bending vibration measurement on rotors using laser technology.

10. J. R. BELL and S. J. ROTHBERG 2000 Journal of Sound and Vibration 238(4), 673-690. Rotational vibration measurements using laser Doppler vibrometry: comprehensive theory and practical application.

11. S. J. ROTHBERG, J. R. BAKER and N. A. HALLIWELL 1989 Journal of Sound and Vibration 135(3), 516-522. Laser vibrometry: pseudo-vibrations. 


\section{LIST OF FIGURES}

Figure 1 - The dual mirror scanning arrangement incorporating two orthogonally aligned mirrors

Figure 2 - Translational and angular misalignment between the scanning system and target rotation axes

Figure 3 - Additional measurement components that occur due to misalignment between the dual mirror scanning system and target rotation axes when employing equal amplitude mirror drive signals $\left(r_{S}=100 \mathrm{~mm}, d_{S}=50 \mathrm{~mm}, z_{0}=1 \mathrm{~m}, x_{0 m}=y_{0 m}=2 \mathrm{~mm}\right.$ and $\left.\theta_{x m}=\theta_{y m}=15 \mathrm{mrad}\right)$

Figure 4 - Experimental validation of the additional measurement component at twice scan frequency which occurs when employing equal amplitude (a) and corrected amplitude (b) mirror drive signals $\left(\Omega_{S}=40 \pi \mathrm{rad} / \mathrm{s}\right)$

Figure 5 - Experimental validation of the additional measurement component at scan frequency for varying $x_{0 m}\left(r_{S}=10 \mathrm{~mm}, d_{S}=50 \mathrm{~mm}, z_{0}=1.272 \mathrm{~m}, \Omega_{S}=20 \pi \mathrm{rad} / \mathrm{s}, y_{0 m}=\right.$ $0 \mathrm{~mm}$ and $\left.\theta_{x m}=\theta_{y m}=0 \mathrm{mrad}\right)$, broken line $=$ initial prediction, solid line $=$ updated prediction

Figure 6 - Initial unknown misalignment analysis using variable $x_{0 m}$ (a) and variable $y_{0 m}$ (b)

Figure 7 - Experimental validation of the additional measurement component at scan frequency for varying $y_{0 m}\left(r_{S}=10 \mathrm{~mm}, d_{S}=50 \mathrm{~mm}, z_{0}=1.272 \mathrm{~m}, \Omega_{S}=20 \pi \mathrm{rad} / \mathrm{s}, x_{0 m}=\right.$ $0 \mathrm{~mm}$ and $\left.\theta_{x m}=\theta_{y m}=0 \mathrm{mrad}\right)$, broken line $=$ initial prediction, solid line $=$ updated prediction

Figure 8 - Experimental validation of the additional measurement component at scan frequency for varying $\theta_{y m}\left(r_{S}=10 \mathrm{~mm}, d_{S}=50 \mathrm{~mm}, z_{0}=1.272 \mathrm{~m}, \Omega_{S}=20 \pi \mathrm{rad} / \mathrm{s}, x_{0 m}=\right.$ 
$y_{0 m}=0 \mathrm{~mm}$ and $\left.\theta_{x m}=0 \mathrm{mrad}\right)$, broken line $=$ initial prediction, solid line $=$ updated prediction

Figure 9 - Experimental validation of the additional measurement component at scan frequency for varying $\Omega\left(r_{S}=10 \mathrm{~mm}, d_{S}=50 \mathrm{~mm}, z_{0}=1.272 \mathrm{~m}, \Omega_{S}=20 \pi \mathrm{rad} / \mathrm{s}, x_{0 m}=y_{0 m}\right.$ $=1 \mathrm{~mm}$ and $\left.\theta_{x m}=\theta_{y m}=0 \mathrm{mrad}\right)$

Figure 10 - Experimental validation of the influence of speckle noise in circular scanning Laser Vibrometer measurements

Figure 11 - Velocity measured by a Laser Vibrometer on a non-rotating target undergoing $40 \mathrm{~Hz}, 10 \mathrm{~mm} / \mathrm{s}$ (nominal) axial vibration

Figure 12 - Velocity measured by a dual mirror circular scanning Laser Vibrometer on a non-rotating target undergoing $40 \mathrm{~Hz}, 10 \mathrm{~mm} / \mathrm{s}$ (nominal) axial vibration $\left(r_{S}=12.5 \mathrm{~mm}\right.$, $d_{S}=50 \mathrm{~mm}, z_{0}=1 \mathrm{~m}, \Omega_{S}=20 \pi \mathrm{rad} / \mathrm{s}$, arbitrary misalignment)

Figure 13 - Velocity measured by a dual mirror circular scanning Laser Vibrometer on a rotating target undergoing $40 \mathrm{~Hz}, 10 \mathrm{~mm} / \mathrm{s}$ (nominal) axial vibration $\left(r_{S}=12.5 \mathrm{~mm}, d_{S}\right.$ $=50 \mathrm{~mm}, z_{0}=1 \mathrm{~m}, \Omega_{S}=20 \pi \mathrm{rad} / \mathrm{s}, \Omega \approx 40 \pi \mathrm{rad} / \mathrm{s}$, arbitrary misalignment)

Figure 14 - Theoretical prediction of the velocity measured by a dual mirror circular scanning Laser Vibrometer on a rotating target undergoing $40 \mathrm{~Hz}, 10 \mathrm{~mm} / \mathrm{s}$ axial vibration $\left(r_{S}=12.5 \mathrm{~mm}, d_{S}=50 \mathrm{~mm}, z_{0}=1 \mathrm{~m}, \Omega_{S}=20 \pi \mathrm{rad} / \mathrm{s}, \Omega=40 \pi \mathrm{rad} / \mathrm{s}, x_{0 m}=y_{0 m}=\right.$ $2 \mathrm{~mm}$ and $\left.\theta_{x m}=\theta_{y m}=5 \mathrm{mrad}\right)$ 


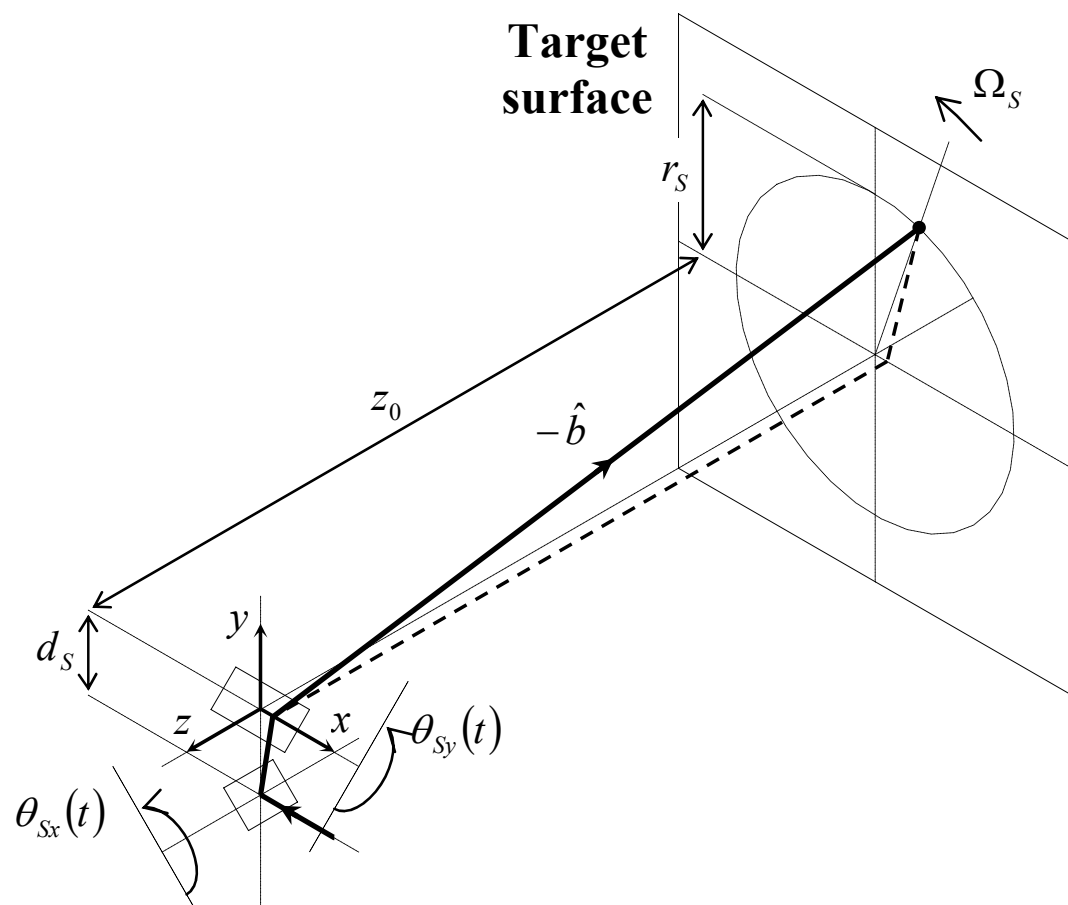

Figure 1 - The dual mirror scanning arrangement incorporating two orthogonally aligned mirrors 

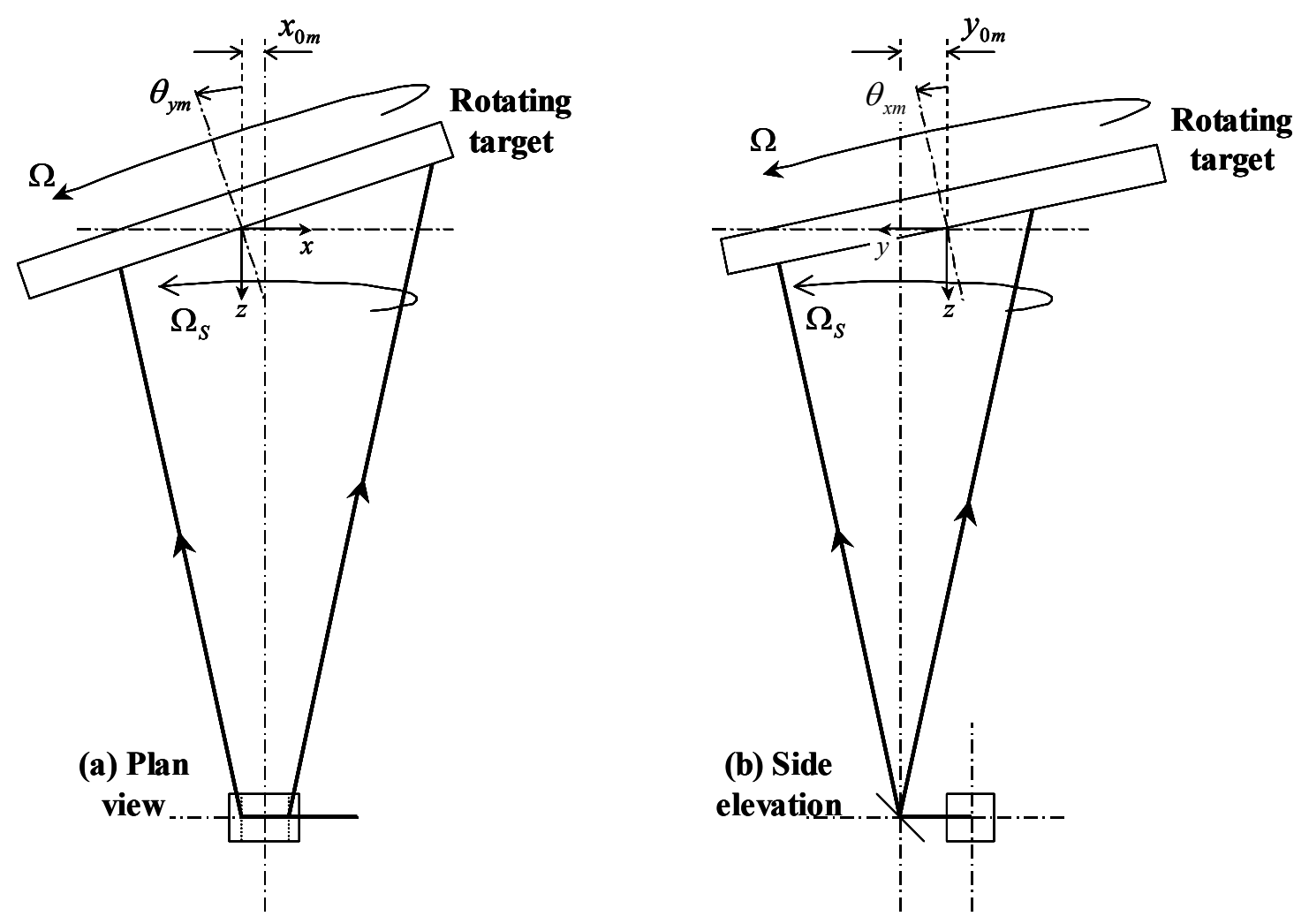

Figure 2 - Translational and angular misalignment between the scanning system and target rotation axes $-x_{0 m}$ and $\theta_{y m}$ only (a) and $y_{0 m}$ and $\theta_{x m}$ only (b)

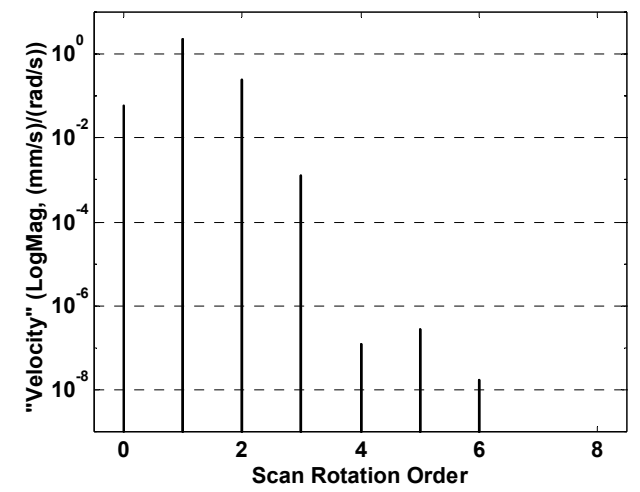

Figure 3 - Additional measurement components that occur due to misalignment between the dual mirror scanning system and target rotation axes when employing equal amplitude mirror drive signals $\left(r_{S}=100 \mathrm{~mm}, d_{S}=50 \mathrm{~mm}, z_{0}=1 \mathrm{~m}, x_{0 m}=y_{0 m}=2 \mathrm{~mm}\right.$ and

$$
\left.\theta_{x m}=\theta_{y m}=15 \mathrm{mrad}\right)
$$



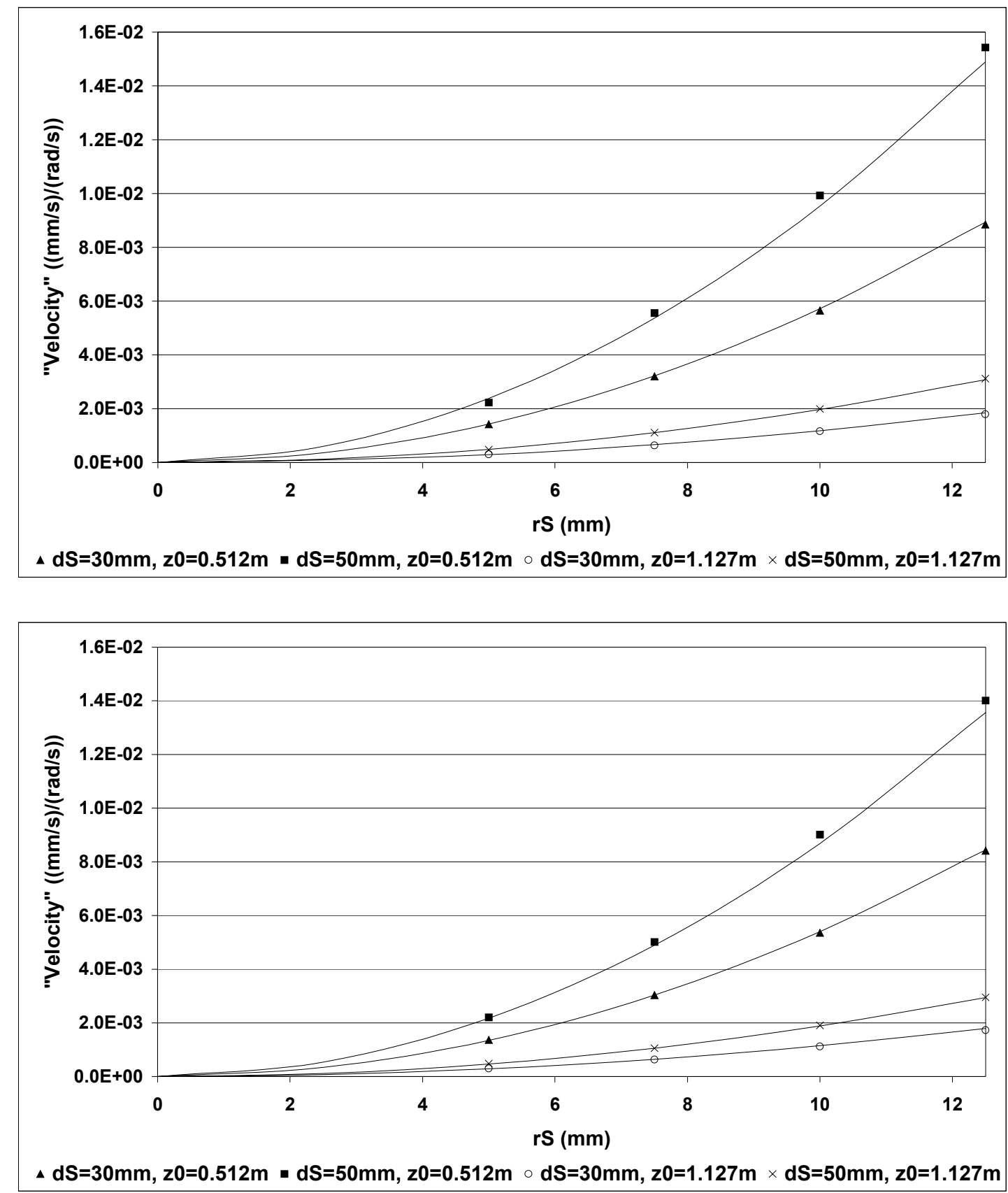

(b)

Figure 4 - Experimental validation of the additional measurement component at twice scan frequency which occurs when employing equal amplitude (a) and corrected amplitude (b) mirror drive signals $\left(\Omega_{S}=40 \pi \mathrm{rad} / \mathrm{s}\right)$ 


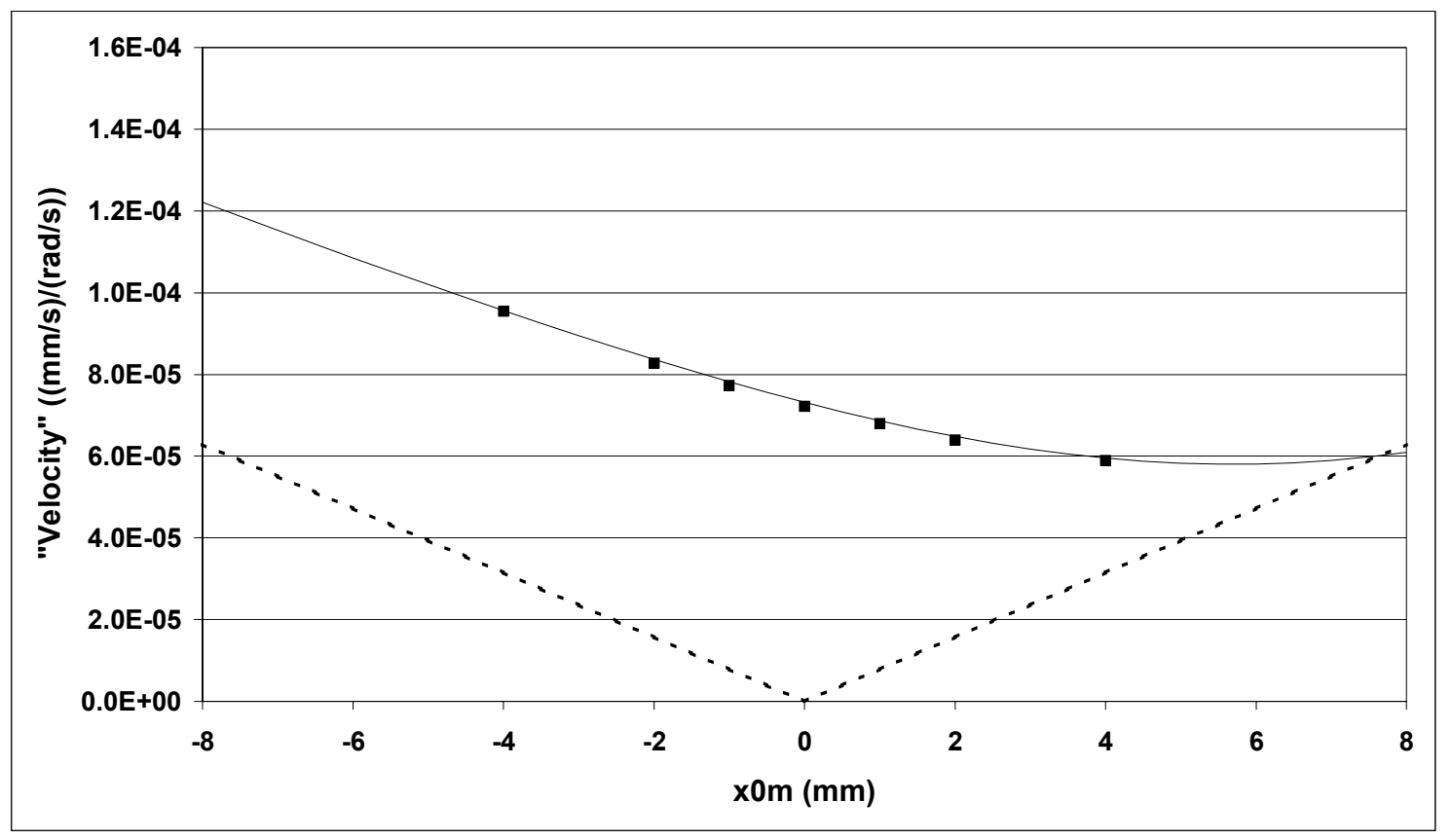

Figure 5 - Experimental validation of the additional measurement component at scan frequency for varying $x_{0 m}\left(r_{S}=10 \mathrm{~mm}, d_{S}=50 \mathrm{~mm}, z_{0}=1.272 \mathrm{~m}, \Omega_{S}=20 \pi \mathrm{rad} / \mathrm{s}, y_{0 m}=\right.$ $0 \mathrm{~mm}$ and $\left.\theta_{x m}=\theta_{y m}=0 \mathrm{mrad}\right)$, broken line $=$ initial prediction, solid line $=$ updated prediction

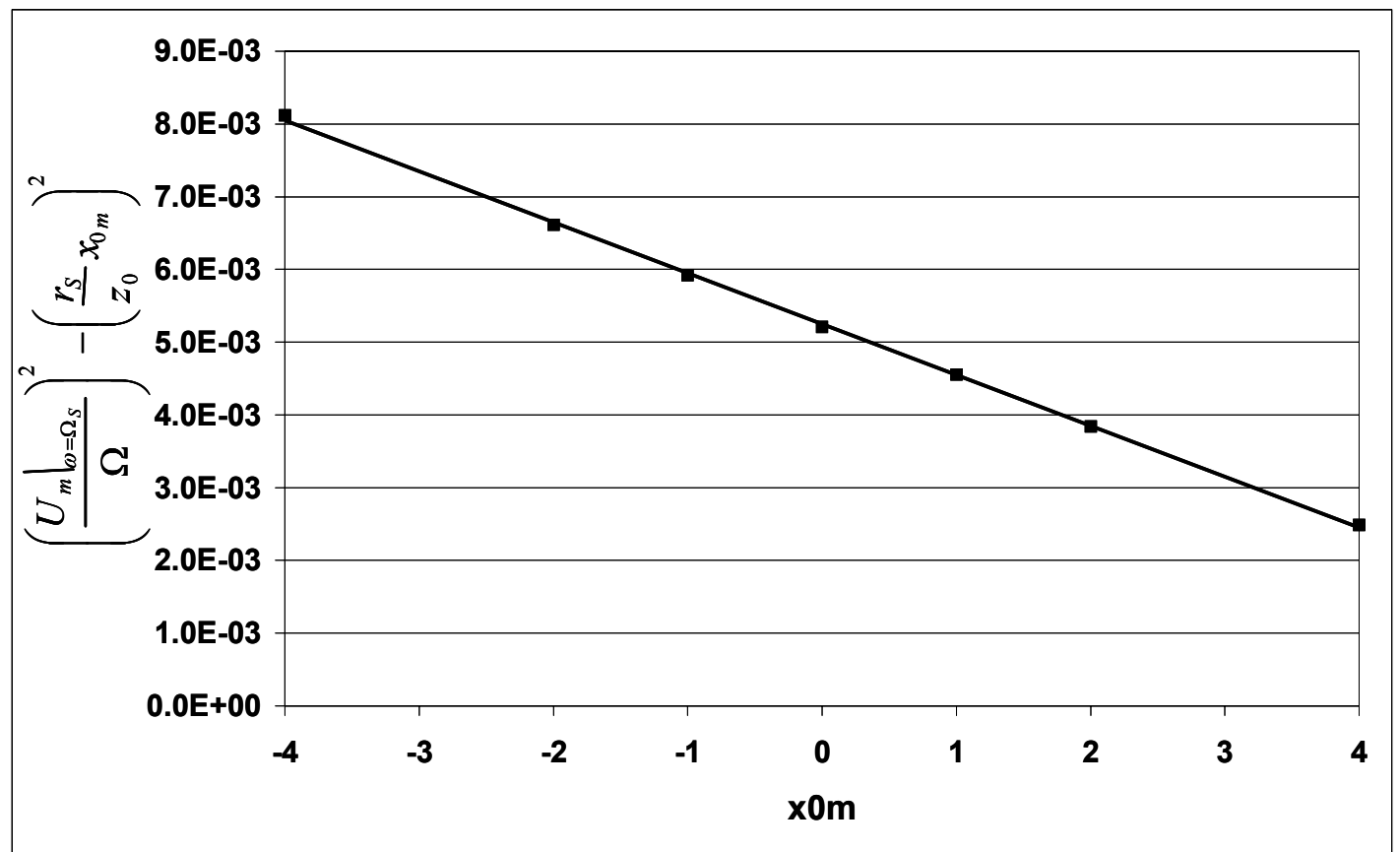

(a) 


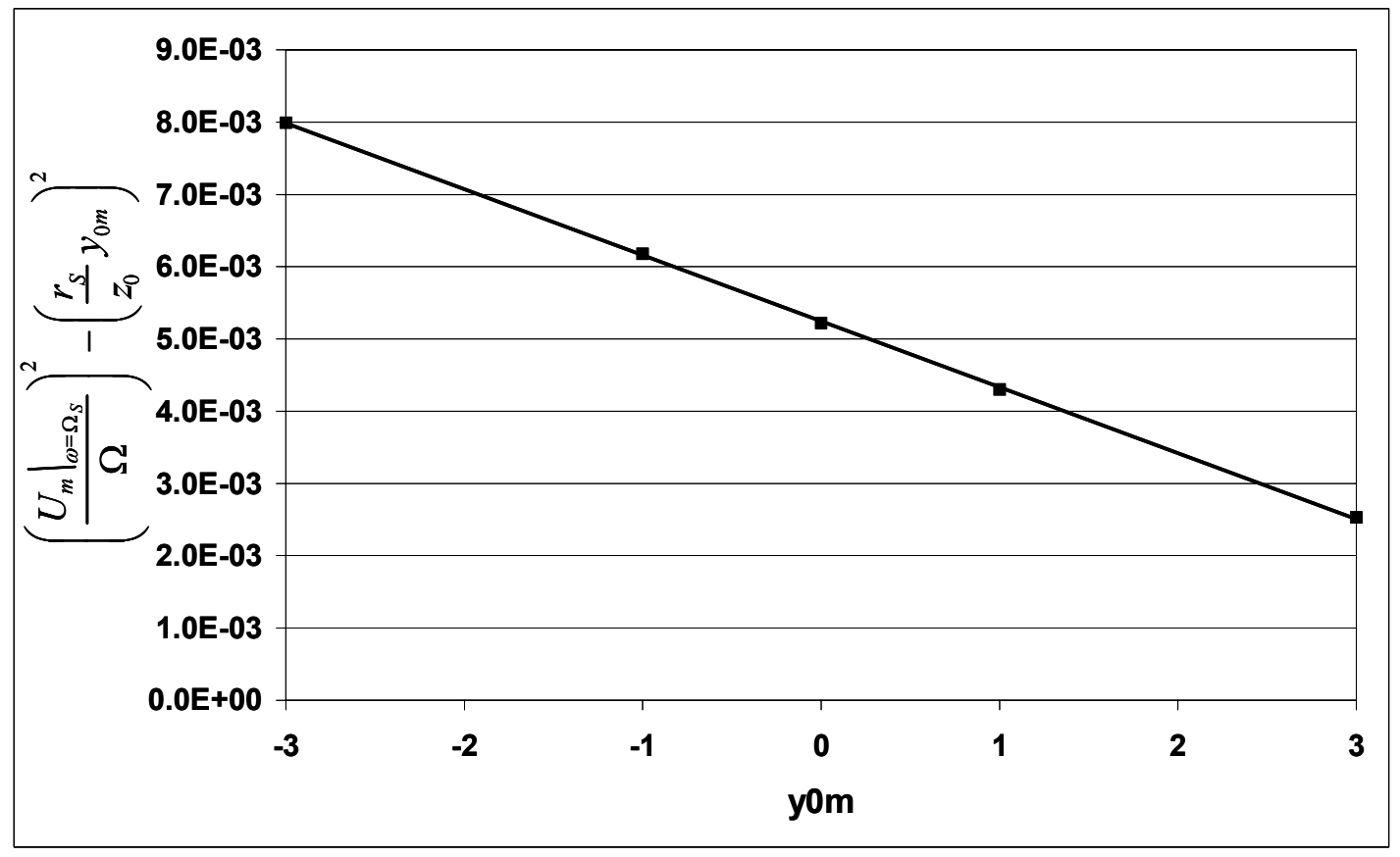

(b)

Figure 6 - Initial unknown misalignment analysis using variable $x_{0 m}$ (a) and variable $y_{0 m}$

(b)

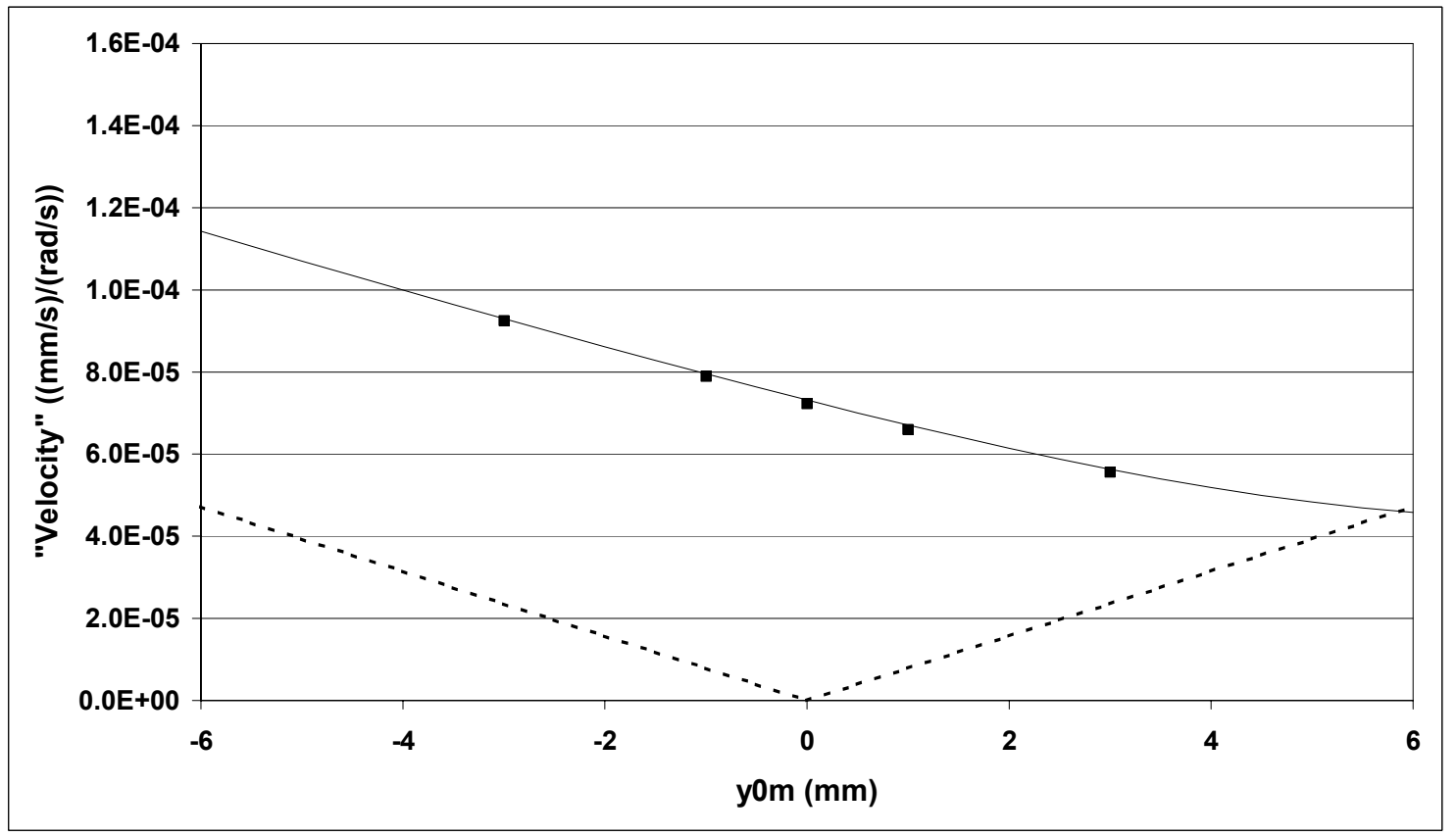

Figure 7 - Experimental validation of the additional measurement component at scan frequency for varying $y_{0 m}\left(r_{S}=10 \mathrm{~mm}, d_{S}=50 \mathrm{~mm}, z_{0}=1.272 \mathrm{~m}, \Omega_{S}=20 \pi \mathrm{rad} / \mathrm{s}, x_{0 m}=\right.$ $0 \mathrm{~mm}$ and $\left.\theta_{x m}=\theta_{y m}=0 \mathrm{mrad}\right)$, broken line $=$ initial prediction, solid line $=$ updated prediction 


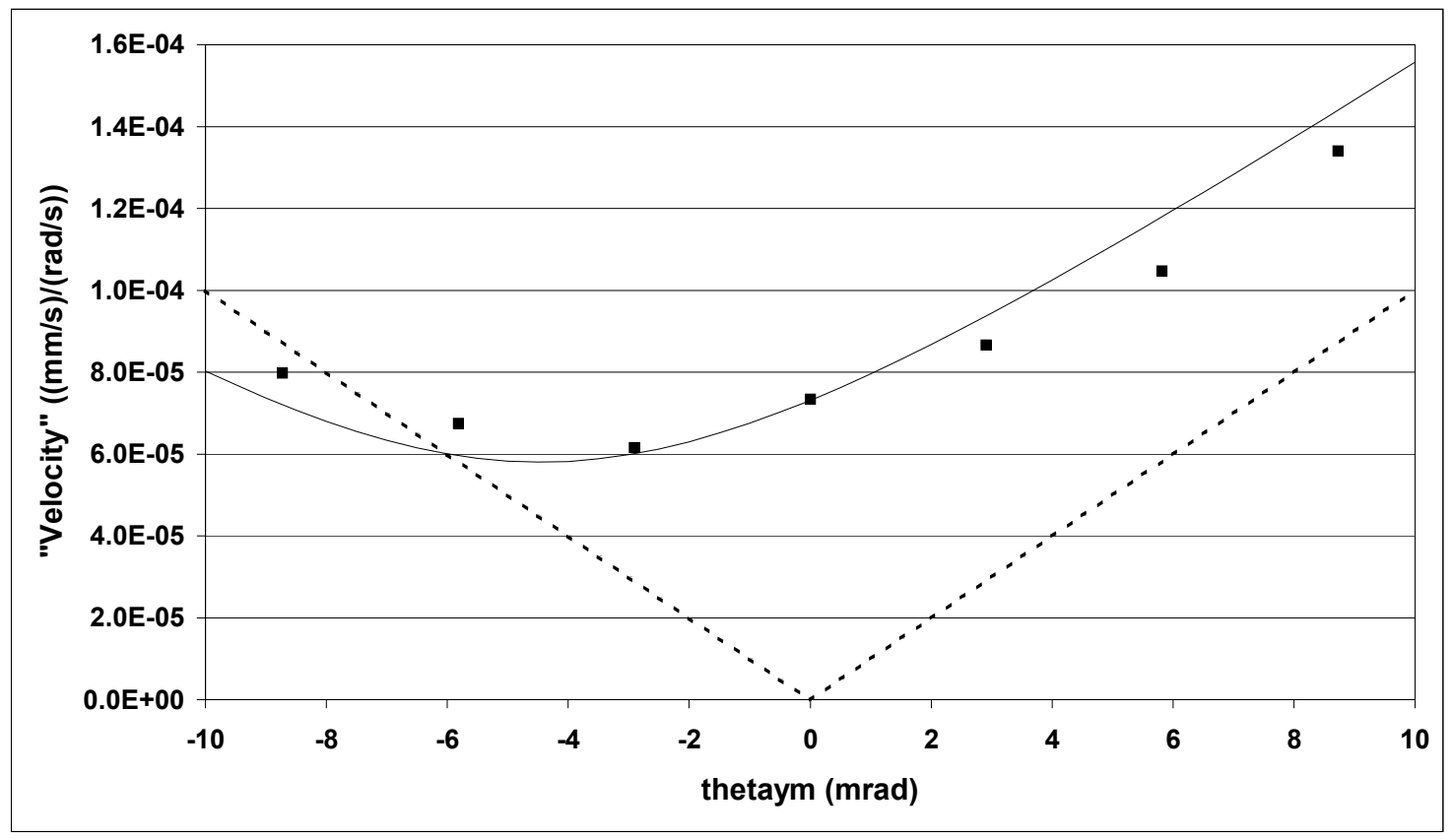

Figure 8 - Experimental validation of the additional measurement component at scan frequency for varying $\theta_{y m}\left(r_{S}=10 \mathrm{~mm}, d_{S}=50 \mathrm{~mm}, z_{0}=1.272 \mathrm{~m}, \Omega_{S}=20 \pi \mathrm{rad} / \mathrm{s}, x_{0 m}=\right.$ $y_{0 m}=0 \mathrm{~mm}$ and $\left.\theta_{x m}=0 \mathrm{mrad}\right)$, broken line $=$ initial prediction, solid line $=$ updated prediction 


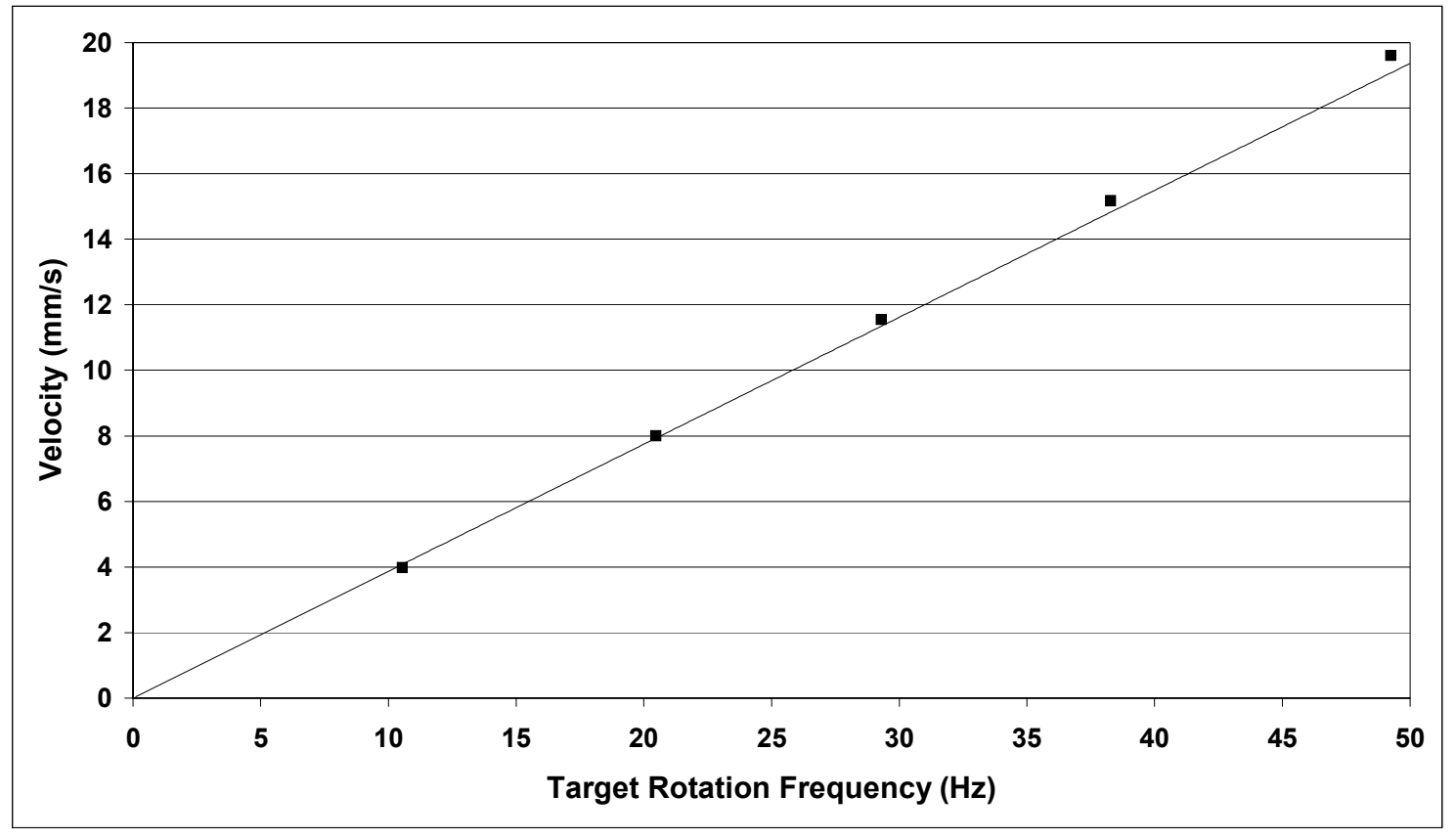

Figure 9 - Experimental validation of the additional measurement component at scan frequency for varying $\Omega\left(r_{S}=10 \mathrm{~mm}, d_{S}=50 \mathrm{~mm}, z_{0}=1.272 \mathrm{~m}, \Omega_{S}=20 \pi \mathrm{rad} / \mathrm{s}, x_{0 m}=y_{0 m}\right.$

$$
\left.=1 \mathrm{~mm} \text { and } \theta_{x m}=\theta_{y m}=0 \mathrm{mrad}\right)
$$

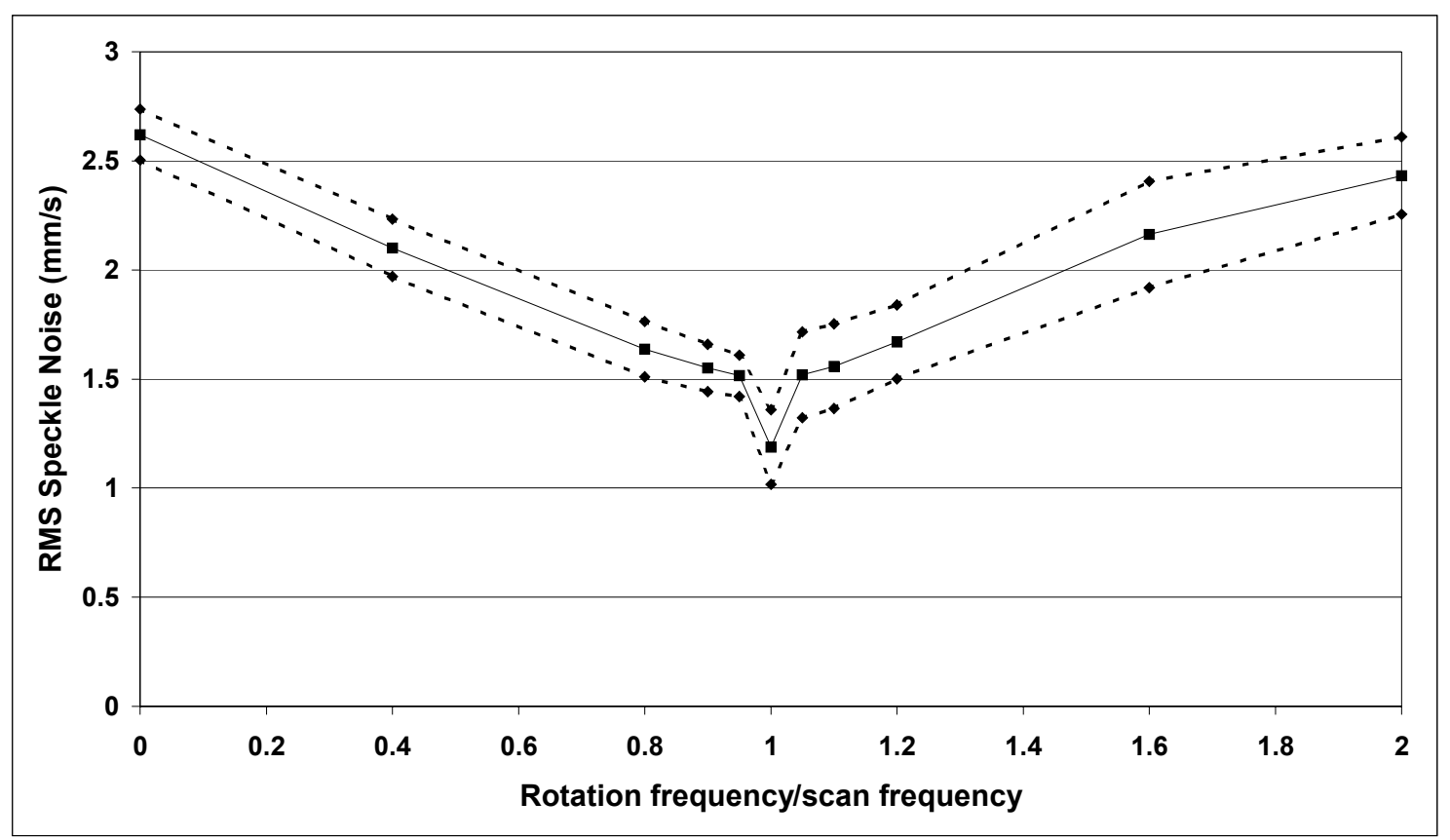

Figure 10 - Experimental validation of the influence of speckle noise in circular scanning Laser Vibrometer measurements 


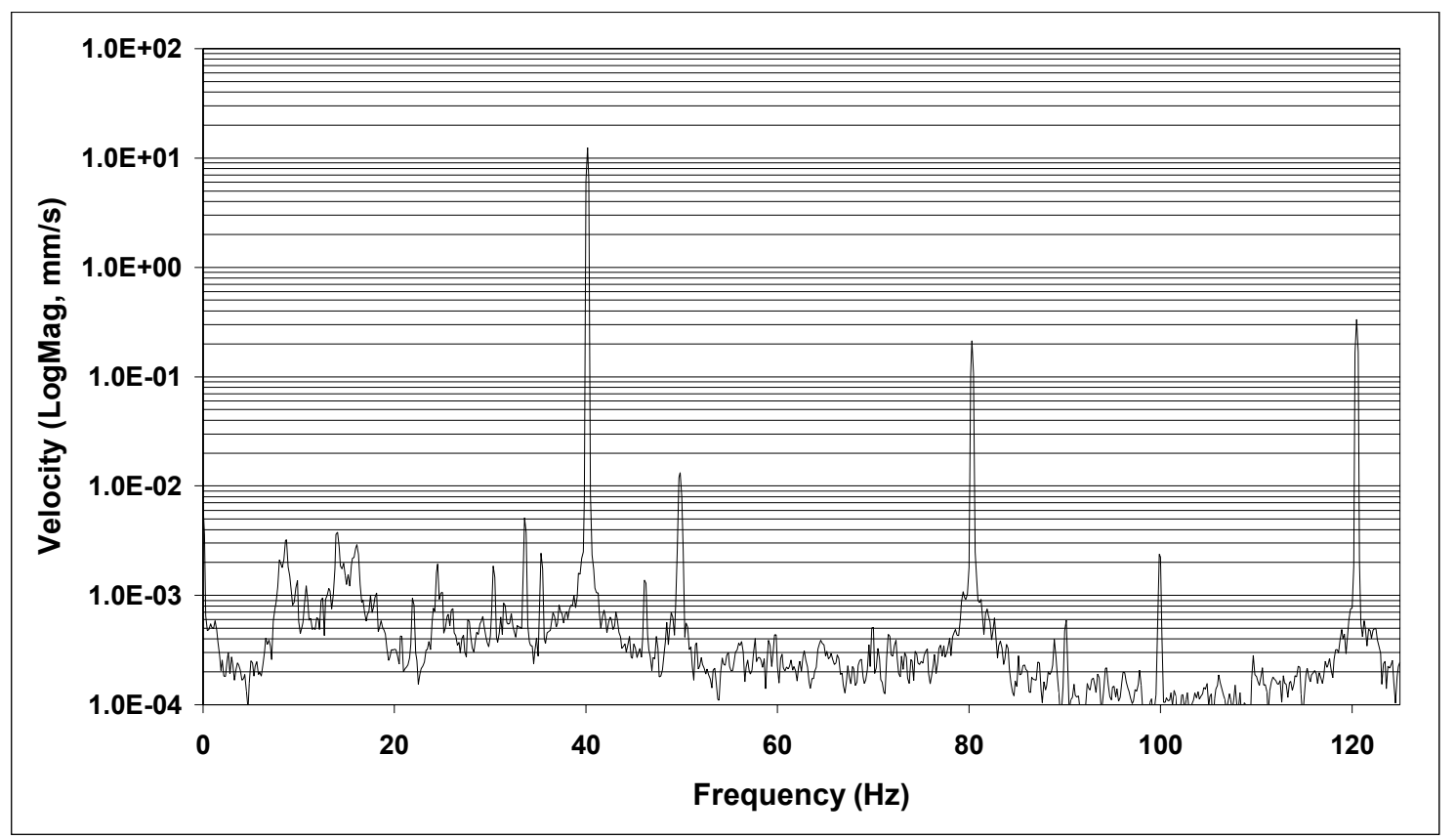

Figure 11 - Velocity measured by a Laser Vibrometer on a non-rotating target undergoing $40 \mathrm{~Hz}, 10 \mathrm{~mm} / \mathrm{s}$ (nominal) axial vibration

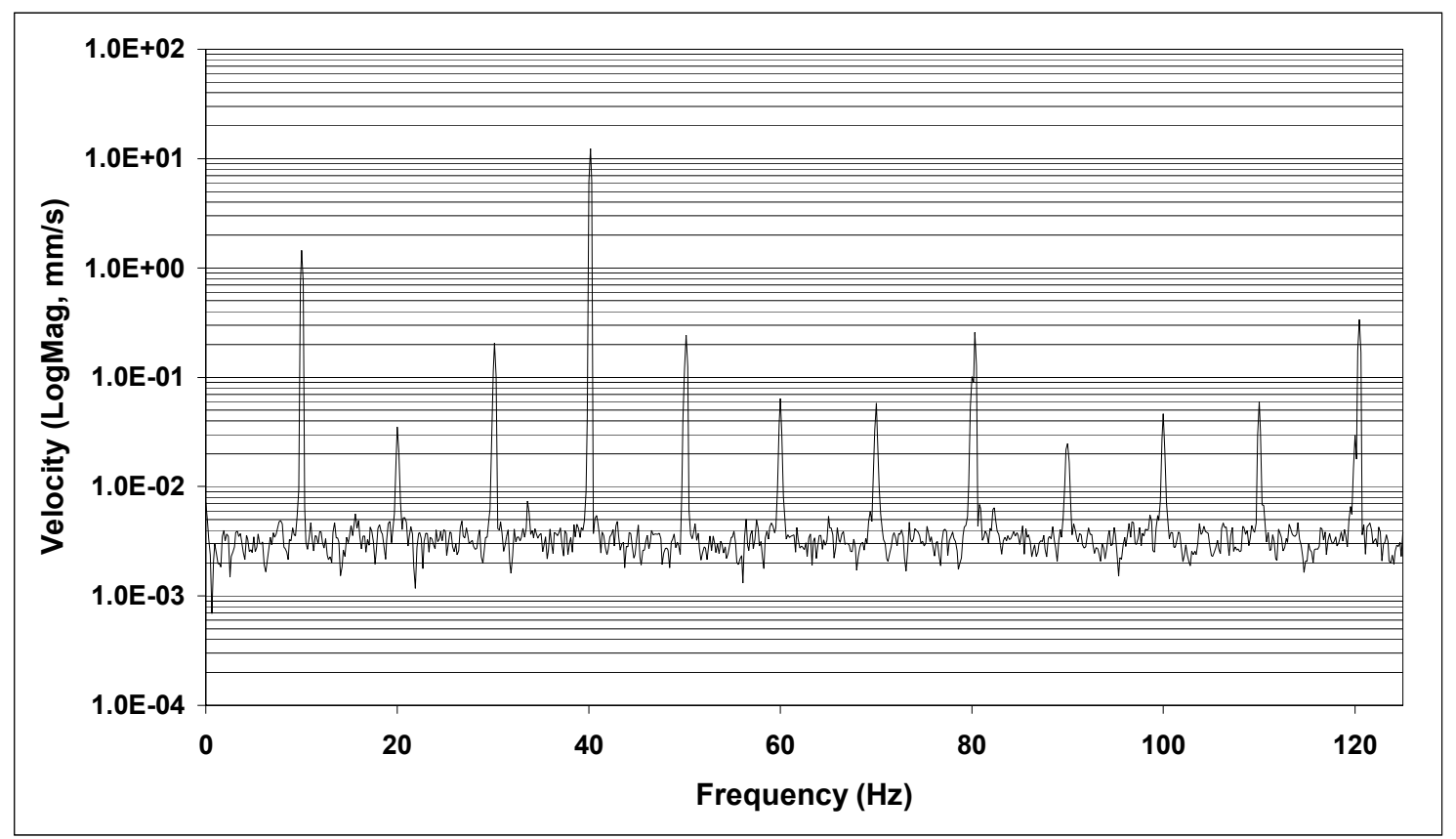

Figure 12 - Velocity measured by a dual mirror circular scanning Laser Vibrometer on a non-rotating target undergoing $40 \mathrm{~Hz}, 10 \mathrm{~mm} / \mathrm{s}$ (nominal) axial vibration $\left(r_{S}=12.5 \mathrm{~mm}\right.$, $d_{S}=50 \mathrm{~mm}, z_{0}=1 \mathrm{~m}, \Omega_{S}=20 \pi \mathrm{rad} / \mathrm{s}$, arbitrary misalignment) 


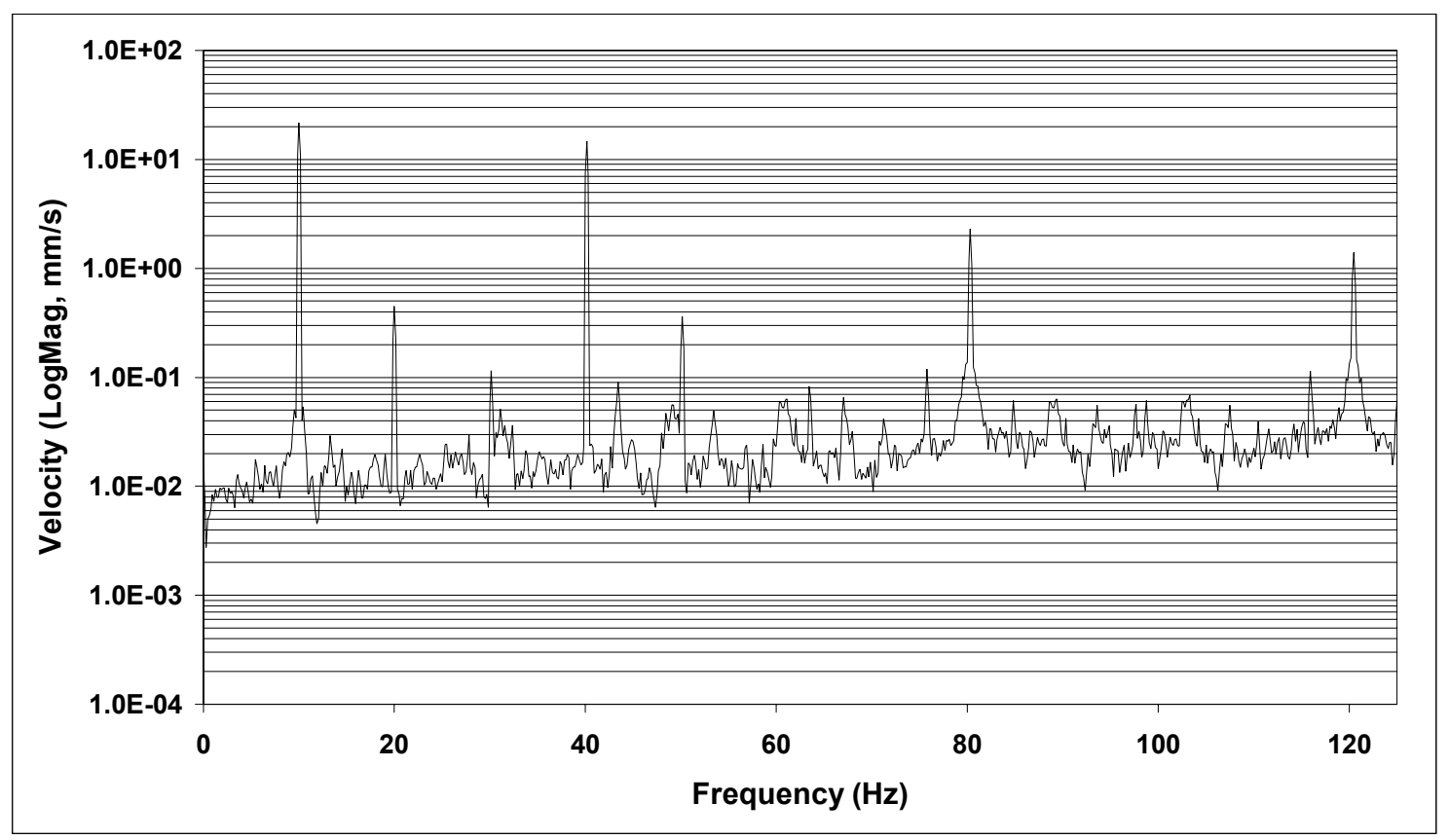

Figure 13 - Velocity measured by a dual mirror circular scanning Laser Vibrometer on a rotating target undergoing $40 \mathrm{~Hz}, 10 \mathrm{~mm} / \mathrm{s}$ (nominal) axial vibration $\left(r_{S}=12.5 \mathrm{~mm}, d_{S}\right.$ $=50 \mathrm{~mm}, z_{0}=1 \mathrm{~m}, \Omega_{S}=20 \pi \mathrm{rad} / \mathrm{s}, \Omega \approx 40 \pi \mathrm{rad} / \mathrm{s}$, arbitrary misalignment)

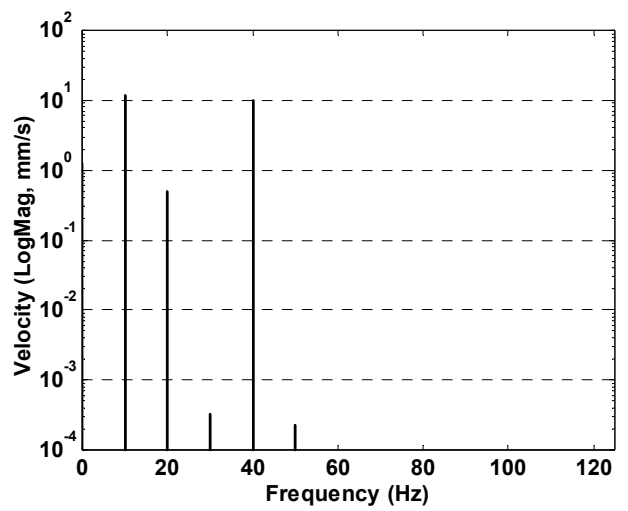

Figure 14 - Theoretical prediction of the velocity measured by a dual mirror circular scanning Laser Vibrometer on a rotating target undergoing $40 \mathrm{~Hz}, 10 \mathrm{~mm} / \mathrm{s}$ axial vibration $\left(r_{S}=12.5 \mathrm{~mm}, d_{S}=50 \mathrm{~mm}, z_{0}=1 \mathrm{~m}, \Omega_{S}=20 \pi \mathrm{rad} / \mathrm{s}, \Omega=40 \pi \mathrm{rad} / \mathrm{s}, x_{0 m}=y_{0 m}=\right.$ $2 \mathrm{~mm}$ and $\left.\theta_{x m}=\theta_{y m}=5 \mathrm{mrad}\right)$ 